\title{
Second-order nonlinear polarizability of various biphenyl derivatives
}

\author{
G. Berkovic, Th. Rasing, and Y. R. Shen \\ Center for Advanced Materials, Lawrence Berkeley Laboratory, Department of Physics, University of California, \\ Berkeley, California 94720 \\ Recoived December 22, 1986; accepted January 13, 1987 \\ The second-order nonlinear polarizabilities of several organic molecules derived from the cyanobiphenyl structure \\ are compared by using second-harmonic generation from molecular monolayers. Changes in optical nonlinearity \\ with changes in molecular structure are discussed.
}

\section{INTRODUCTION}

The current interest in finding organic materials having very large optical nonlinearities is a result of the promising potential applications of such materials in a wide variety of optoelectronic devices. ${ }^{1}$ Finding suitable organic materials for such applications will require a fundamental understanding of the connection between molecular. structure and optical nonlinearity. In addition, it is necessary to have a convenient and reliable method for determining the nonlinearity of a molecule or material.

Second-order nonlinear optical constants are usually determined by one of the following methods. ${ }^{2}$ If the material crystallizes in a noncentrosymmetric structure, the nonlinearity can be determined from a direct measurement of the second-harmonic generation (SHG) from a single crystal. In the absence of a sufficiently large single crystal, the non: linearity may be determined by comparing the SHG of a powdered sample with that of a standard powdered nonlinear material. ${ }^{3}$ Molecular nonlinearities, on the other hand, are usually determined by dc electric-field-induced secondharmonic generation (EFISH) in solution. ${ }^{2,4}$ A shortcoming of this method is that in this case SHG is caused by both the second-order $\left[\ddot{\alpha}^{(2)}\right]$ and the third-order $\left[\ddot{\alpha}^{(3)}\right]$ polarizabilities of the molecule, and thus, in order to evaluate $\ddot{\alpha}^{(2)}$ accurate. ly, a good knowledge of solvent-solute interactions and locel

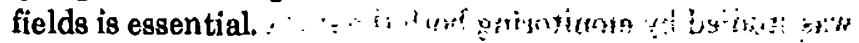

Recently we have suggested an alternative method for determination of second-order molecular polarizabilities. ${ }^{5}$ In this method the material under study is spread as a monomolecular layer on a suitable substrate (such as roter), and $\vec{\alpha}^{(2)}$ is determined by the SHG from this interface. Bicause of its symmetry requirements SHG is an inherently surface-sensitive process, and, when the molecular nonlinearity is sufficiently large, the contribution from the monolayer should be dominant or at least easily extractable from the total signal.5,6 This technique has the advantages of a low background signal and an easily controllable surface density of molecules. In earlier studies, $\vec{\alpha}^{(2)}$ of molecules on glass was determined by SHG from a monolayer either spread on glass ${ }^{7,8}$ or adsorbed by the Langmuir-Blodgett

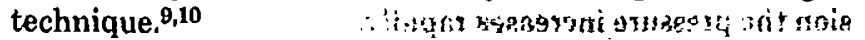

In this paper we use the monolayer method to measure $\vec{\alpha}^{(2)}$ for a series of structurally similar molecules starting from a cyanobiphenyl, which we have shown to be highly nonlinear. ${ }^{5}$ The molecules studied are shown in Fig. 1 . Studying this set of molecules enables us to determine the change in $\vec{\alpha}^{(2)}$ resulting from the following changes in molecular structure: lengthening the hydrocarbon tail; replacement of the cyano group by a carboxylic acid group; replacing one phenyl ring with a pyrimidine ring; and adding a third phenyl ring.

We have measured the second-order nonlinear polarizability $\ddot{\alpha}^{(2)}(2 \omega ; \omega, \omega)$ at frequencies in which $2 \omega$ is resonant or near resonant with a molecular transition. At these frequencies, there is a significant resonant enhancement of $\vec{\alpha}^{(2)}$ without laser-induced damage or desorption, unlike in the case when $\omega$ is near resonance.

\section{EXPERIMENT}

Monolayers of the materials under study were spread on a water surface in a Langmuir trough by pipetting an appropriate volume of a solution (in hexane, petroleum ether, or 4:1 hexane:trichloroethylene) and waiting a sufficient time for the solvent to evaporate and the monolayer to equilibrate (see also below).

SHG measurements were performed by focusing laser irradiation of frequency $\omega$ on the water surface at an incident angle of $60^{\circ}$ and detecting photons of frequency $2 \omega$ in the reflected output. ${ }^{6}$ For most experiments we utilized the frequency-doubled output (532 nm) of a $500-\mathrm{Hz} Q$-switched $\mathrm{Nd}$ :YAG laser in which each $Q$-switch envelope contained approximately 10 mode-locked pulses of 50 -psec duration. At full laser power, the total energy at the water surface was $0.4 \mathrm{~mJ}$ per $Q$-switch pulse, For frequency tuning we used a Nd:YAG-pumped dye laser giving 10 -nsec pulses at $10 \mathrm{~Hz}$. The maximum dye-laser power at the sample was $6 \mathrm{~mJ} /$ pulse for 586-nm excitation and aproximately $1-\mathrm{mJ} /$ pulse for excitation in the region $510-560 \mathrm{~nm}$. For accurate determination of relative SHG intensities, a photon-counting detection scheme was used. Reliable photon-counting statistics were ensured by either reducing the input laser power or attenuating the output transmission for light of frequency $2 \omega$, in order to give an average output signal of no more than 0.2 photon count/pulse (which was averaged over at least $6 \times$ $10^{3}$ pulses).

The second-harmonic signal in the reflected direction is given by ${ }^{5,8}$ 

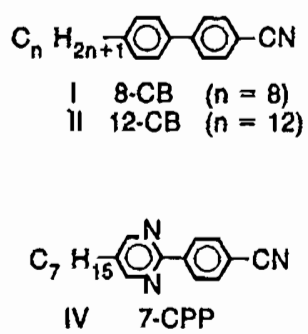

IV $\quad 7-\mathrm{CPP}$
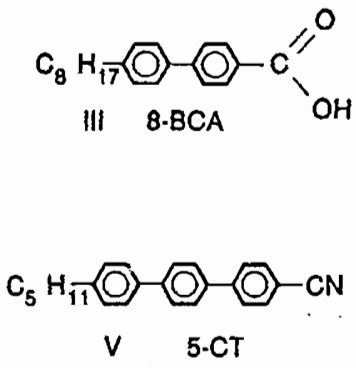

Fig. 1. Materials used in this study:

\begin{tabular}{cccc} 
Number & Abbreviation & Full Name & $\begin{array}{c}\text { Alternative } \\
\text { Name }\end{array}$ \\
\hline I & 8CB & $\begin{array}{c}4^{\prime} \text {-n-octyl-4-cyanobi- } \\
\text { phenyl }\end{array}$ & K-24 \\
II & 12CB & $\begin{array}{c}4^{\prime} \text {-n-dodecyl-4- } \\
\text { cyanobiphenyl }\end{array}$ & K-36 \\
III & 8BCA & $\begin{array}{c}4^{\prime} \text {-n-ocytl-biphenyl- } \\
\text { 4-carboxylic acid }\end{array}$ \\
IV & 7CPP & $\begin{array}{c}\text { 6-n-heptyl-2-(4-cyano- } \\
\text { phenyl)pyrimidine } \\
4^{\prime \prime} \text {-n-pentyl-4-cyano- } \\
\text {-p-terphenyl }\end{array}$ & $\mathrm{P}_{3}-7$ \\
V & 5CT & T-15
\end{tabular}

Alternative names are the manufacturers' nomenclature, but they are not used further in this paper.

$$
I(2 \omega)=\frac{32 \pi^{3} \omega^{2}}{c^{3} \epsilon(\omega) \epsilon^{1 / 2}(2 \omega)}\left|\mathbf{e}_{2 \omega} \vec{\chi}_{s}^{(2)}: \mathbf{e}_{\omega} \mathbf{e}_{\omega}\right|^{2} I^{2}(\omega)
$$

where $e_{\Omega}=L_{\Omega} \hat{e}_{\Omega}$, with $\hat{e}_{\Omega}$ the unit polarization vector of the field at frequency $\Omega$ and $L_{n}$ the Fresnel factor of the field; $\epsilon(\omega)$ is the substrate dielectric constant; $I(\omega)$ is the laser intensity; and $\tilde{\chi}_{s}{ }^{(2)}$ is the second-order surface-susceptibility tensor.

The contributions to $\ddot{\chi}_{8}{ }^{(2)}$ may be represented as

$$
\ddot{\chi}_{s}^{(2)}=\ddot{\chi}_{w}^{(2)}+\ddot{\chi}_{m}^{(2)}+\ddot{\chi}_{\text {int }}^{(2)}
$$

where $\ddot{\chi} w^{(2)}$ and $\ddot{\chi}_{m}{ }^{(2)}$ are the susceptibilities of the substrate (water) and adsorbate monolayer, respectively, and $\bar{\chi}_{\text {nt }}^{(2)}$ includes any perturbational interaction between them. For an isotropic substrate such as water, the second-order susceptibility is electric dipole forbidden in the bulk, and $\vec{\chi}_{w}{ }^{(2)}$ is expected to be weak, arising mainly from a weak quadru. pole effect. ${ }^{11}$ For adsorbates of moderate nonlinearity the SHG signal from an adsorbate-covered surface far exceeds that of the bare surface, and in such cases we may approximate $\bar{\chi}_{s}^{(2)}=\bar{\chi}_{m}{ }^{(2)}$.

In order to obtain the components of the polarizability tensor $\vec{\chi}^{(2)}$ of individual molecules from the surface-susceptibility tensor $\bar{\chi}^{(2)}$, the spatial transformation $\left\langle T_{i j k}^{\lambda \mu \nu}\right\rangle$ from the laboratory $(i j k)$ axes to the molecular $(\lambda \mu \nu)$ axes must generally be known. However, in the case when $\ddot{\alpha}^{(2)}$ is dominated by a single component $\alpha_{\xi \xi \xi}^{(2)}$ along a certain molecular axis $\xi$, and the latter is randomly distributed around the surface normal, the situation is greatly simplified. The nonvanishing elements of $\ddot{\chi}_{m}^{(2)}$ are then directly proportional to $\alpha_{\xi \xi \xi}^{(2)}$, assuming that the interaction between molecules is neg. ligible. ${ }^{8}$

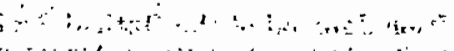

$$
\begin{aligned}
& \chi_{8, z 2 z}^{(2)}=N_{s}\left\langle\cos ^{3} \theta\right\rangle \alpha_{\xi \xi \xi}^{(2)} \\
& \dot{\chi}_{s, 2 i i}^{(2)}=\dot{\chi}_{s, 3 i}^{(2)}=\chi_{s, 1 i z}^{(2)}=\frac{1}{2} N_{s}\left\langle\sin ^{2} \theta \cos \theta\right\rangle \alpha_{\xi \xi \xi}^{(2)}
\end{aligned}
$$

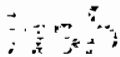

$$
\begin{aligned}
& i=x, y,
\end{aligned}
$$

Berkovic et al.

where $\theta$ is the polar angle between $\hat{\xi}$ and the surface normal $\hat{z}$, and $N_{s}$ is the surface density of adsorbate molecules. From the above equations, it follows that a measurement of the ratio of any two linear combinations of $\chi_{8,22 z}^{(2)}$ and $\chi_{s, z i i}^{(2)}$ will yield a weighted average of $\theta$.

For an orientationally ordered monolayer of amphiphilic molecules on water, the orientational distribution is expected to be sharply peaked for a certain value of $\theta$. Approximating the distribution by a $\delta$ function, we can then find the value of $\theta$ from the ratio of $\chi_{s, z z z}^{(2)}$ and $\chi_{s, z i i}^{(2)}$, which then yields $\alpha(2)$

\section{RESULTS}

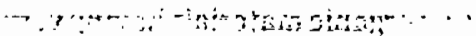

Spreading Characteristics of Molecules on Water

Our measurements were performed on molecular monolayers spread on water. The molecules chosen all have a nonlinear functional group incorporated in an amphiphilic structure, i.e., a hydrophilic (polar) head and a hydrophobic tail. Whether such a molecule will spread on the water surface depends on the difference between $W_{A}$, the work of adhesion of the molecule to water, and $W_{c}$, the work of cohesion between the molecules. One can define a spreading coefficient $S$ as ${ }_{-}^{12}$ isinit

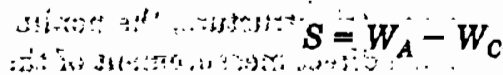

No material will spread if $S<0$, as supported by experimental observation. If $S>0$ the molecule will spread, although this condition alone does not ensure formation of monolayers (e.g., the molecule may be more stable in multilayers). When monolayer formation occurs, the spreading coefficient $S$ is equal to the equilibrium spreading pressure $\pi_{e}$, which can be measured by putting a small amount of the bulk material on the water surface and measuring the change in the surface tension of water. ${ }^{12}$. Thus, if $\pi_{e}>0$, the molecules can be spread from solution to give a monolayer on water.

. The monolayer formation of molecules used in this work was studied by monitoring both the surface tension and the SHG signal after spreading the molecules from solution. For $8 C B, 12 C B$, and $5 C T$ the surface tension would reach a stable value in approximately $10 \mathrm{~min}$, the time that it takes for the solvent to evaporate fully. The stability of the surface tension afterward indicates that the average surface density does not change and that the molecules stay at the surface. Pressure-area curves for these molecules are presented in Fig. 2. Both $8 \mathrm{CB}$ and $12 \mathrm{CB}$ exhibit a similar phase diagram in which the surface pressure is practically constant for areas between 25 and $40 \AA^{2} /$ molecule. The lower surface pressure of $12 \mathrm{CB}$ in this phase is consistent with its larger mass. ${ }^{13}$. On the other hand, $5 \mathrm{CT}$ shows a different type of behavior, exerting pressure only as the molecules become very close packed ( $\leq 25 \AA^{2} /$ molecule); with further compression the pressure increases rapidly.

Although when monolayers of these materials were spread on water a stable gurface pressure was quickly achieved, the 


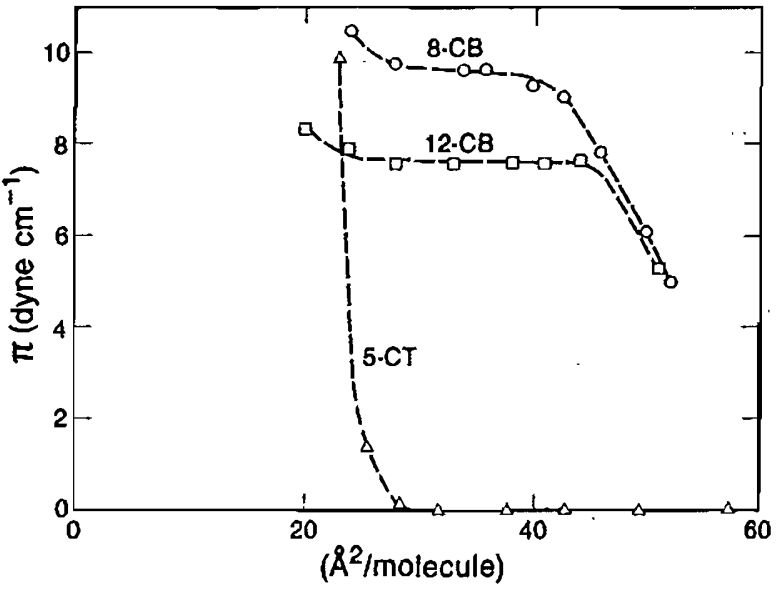

Fig. 2. Surface pressure $(\pi)$ versus area per molecule for $8 \mathrm{CB}$, $12 \mathrm{CB}$, and 5CT spread on water.

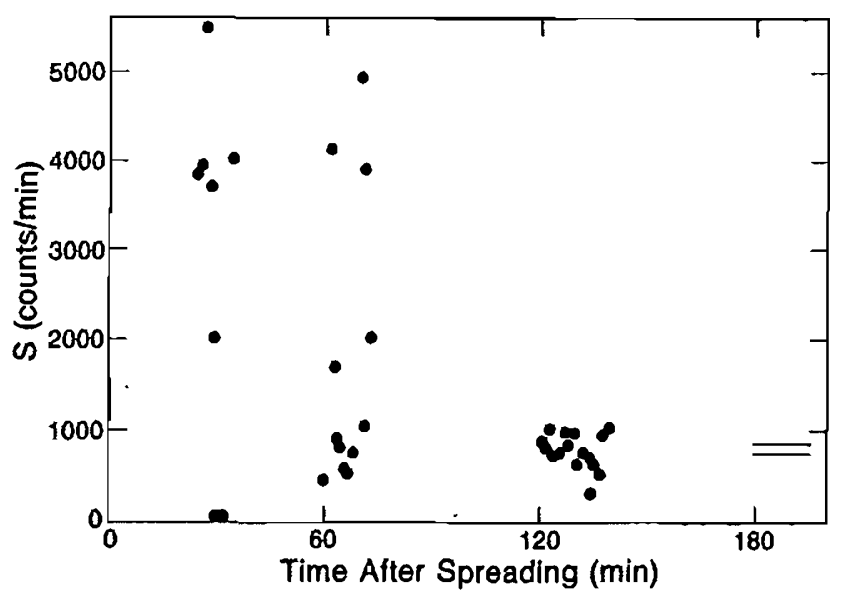

Fig. 3. SHG signals (number of counts per minute) recorded at various times after spreading $12 \mathrm{CB}$ on water at a density of $60 \mathrm{~A}^{2} /$ molecule. After $3 \mathrm{~h}$ the SHG signal was stable and reproducible, as 20 successive readings all fell between the two lines indicated.

SHG signal took longer to stabilize. Fluctuations in the SHG signal observed for $12 \mathrm{CB}$ spread at $60 \AA^{2} /$ molecule are shown in Fig. 3. Analysis of the data shows that there are large fluctuations in both density and orientation of the molecules that disappear only after several hours (see Fig. 3). This behavior arises from the fact that, by the nature of the spreading technique, initially all the molecules are randomly distributed on the surface. However, in the absence of any phase transitions, there is only one thermodynamically stable configuration for a given temperature and pressure, and the monolayer will slowly organize itself into a homoge. neous configuration. Because the molecules are constrained to the surface plane, and because stirring occurs only through the slow convective motion of the water, this self-diffusion takes a long time.

The stable SHG signals observed at long times for $8 \mathrm{CB}$, $12 \mathrm{CB}$, and $5 \mathrm{CT}$ were reproducible and thus considered to be the true signal associated with the corresponding equilibrated monolayer and were used in the evaluation of $\alpha^{(2)}$ detailed below.

For $8 \mathrm{BCA}$ and $7 \mathrm{CPP}, \pi_{e} \simeq 0$, and no stable monolayers should be obtainable on a water surface. In fact, we ob. served that, after spreading, both the surface pressure and the SHG signal gradually disappeared with time. This can be attributed to the material's dissolving into the water subphase. From absorption spectra of saturated aqueous solutions of these materials, we estimate their solubilities to be $200 \mu \mathrm{g} / \mathrm{L}$ (7CPP) and $60 \mu \mathrm{g} / \mathrm{L}(8 \mathrm{BCA})$. This very low solubility is indeed sufficient to prevent monolayer formation because in a typical experiment $20 \mu \mathrm{g}$ of material is spread on a trough of surface area $130 \mathrm{~cm}^{2}$ and volume $0.7 \mathrm{~L}$.

Nevertheless, we were able to make monolayers of $8 \mathrm{BCA}$ and 7CPP showing stable SHG signals. This was achieved by presaturating the subphase with the adsorbate and then adding an additional amount of the adsorbate to give a monolayer of the desired surface density. In addition, for $8 \mathrm{BCA}$ the subphase was made slightly basic by the addition of a small volume of ammonia solution. Samples prepared in this way gave stable SHG signals that were of the same magnitude as the initial SHG signals observed when these materials were spread on pure aqueous subphases. As a further check of the validity of the monolayers prepared in this way, we compared their SHG signals with those of samples prepared by spin coating material onto glass slides. Although the magnitude of the signal from the latter sam. ples showed some variation from spot to spot on the slides, they were essentially the same as the SHG signals of the monolayers spread on presaturated aqueous substrates.

In order to determine the bulk aqueous solubility of the other materials that apparently do stay at the water surface, we attempted to find evidence of $12 \mathrm{CB}$ in the aqueous phase by stirring $12 \mathrm{CB}$ crystals in water for several days and, alternatively, by checking the subphase after a 12CB monolayer was left on the water surface for $48 \mathrm{~h}$. In both cases, no $12 \mathrm{CB}$ could be detected in the aqueous phase, indicating that its solubility is less than the detection limit $(5 \mu \mathrm{g} / \mathrm{L})$.

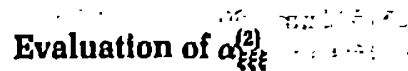

-When monolayers were prepared as described in the preceding subsection, stable SHG signals were realized. Equations (1) and (3) show that, in the absence of local-field effects, the square root of the SHG signal is proportional to $\chi^{(2)}$ and thus

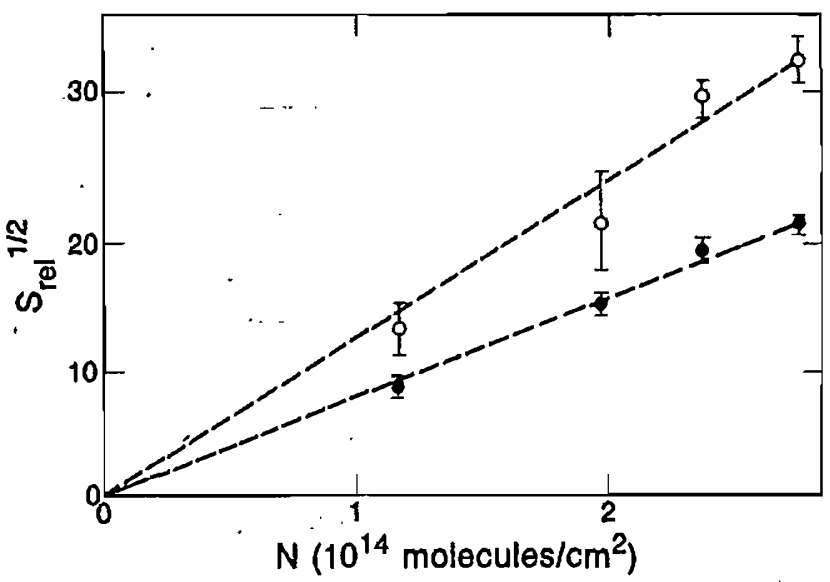

Fig. 4. The square root of the relative SHG intensity $\left(S_{\text {ril }}^{1 / 2}\right)$ is plotted as a function of surface density for $5 C T$. The input laser field was polarized at $45^{\circ}$ to the plane of incidence, for which both the s-polarized (open circles) and $p$-polarized (filled circles) SHG outputs were measured. 
to $N_{s}$, the surface density of adsorbate molecules. As shown in Fig. 4, this proportionality was found to hold for surface densities up to $N=3 \times 10^{14} \mathrm{~cm}^{-2}\left(33 \AA^{2} /\right.$ molecule $)$.

To evaluate $\alpha_{k \xi \xi}^{(2)}$, we measured SHG from all these monolayers under identical geometrical conditions. The two nonvanishing components of $\ddot{\chi}_{s}{ }^{(2)}$ were determined from the s- and p-polarized SHG output intensities following $45^{\circ}$ polarized incident excitation and the appropriate Fresnel coefficients. The magnitudes of $\chi$ were calibrated against the pure-water SHG signal, for which $\chi_{y z y}^{(2)}=2 \times 10^{-17}$ esu. ${ }^{5}$ The assumptions in Eqs. (1)-(3) were verified by the facts that the SHG signal is proportional to $N_{s}^{2}$ for the surface densities used; that the SHG signal from all adsorbate-covered water surfaces was $90-3000$ times that of pure water; and that by using different input and output polarizations we could show that $\chi_{s, z y y}^{(2)}=\chi_{s, y z y}^{(2)}$. From the values of $\chi^{(2)}, \theta$ and $\alpha_{\xi \xi \xi}^{(2)}$ are readily calculated, as summarized in Table 1 .

Four of the compounds studied, $8 \mathrm{CB}, 12 \mathrm{CB}, 8 \mathrm{BCA}$, and $7 \mathrm{CPP}$, all have solution absorption maxima (in $n$-hexane) very close to $266 \mathrm{~nm}$ (see Fig. 5), and thus there should be some $2 \omega$ resonant enhancement for SHG of $532-\mathrm{nm}$ excitation. For a valid comparison of $\alpha^{(2)}$ values, SHG for 5CT

Table 1. Surface Susceptibilities, Tilt Angle, and Second-Order Molecular Polarizability for Molecules I- $\mathrm{V}^{\mathrm{a}}$

\begin{tabular}{clcc}
\hline Molecule & $\begin{array}{c}\chi_{y z y} \\
\left(10^{-16} \text { esu }\right)^{6}\end{array}$ & $\theta$ & $\begin{array}{c}\alpha_{k \xi \xi}^{(2)} \\
\left(10^{-30} \text { esu }\right)\end{array}$ \\
\hline $8 \mathrm{CB}=\mathrm{C}_{8} \mathrm{H}_{17}\left(\mathrm{C}_{6} \mathrm{H}_{4}\right)_{2} \mathrm{CN}$ & 11 & 71 & 25 \\
$12 \mathrm{CB}=\mathrm{C}_{12} \mathrm{H}_{25}\left(\mathrm{C}_{6} \mathrm{H}_{4}\right)_{2} \mathrm{CN}$ & 11 & 71 & 25 \\
$8 \mathrm{BCA}=\mathrm{C}_{8} \mathrm{H}_{17}\left(\mathrm{C}_{6} \mathrm{H}_{4}\right)_{2} \mathrm{COOH}$ & 2.8 & 63 & 6 \\
$7 \mathrm{CPP}=$ & 1.9 & 79 & 8 \\
$\mathrm{C}_{7} \mathrm{H}_{15}\left(\mathrm{C}_{4} \mathrm{~N}_{2} \mathrm{H}_{2}\right) \mathrm{C}_{6} \mathrm{H}_{4} \mathrm{CN}$ & & \multicolumn{2}{c}{} \\
$5 \mathrm{CT}=\mathrm{C}_{5} \mathrm{H}_{11}\left(\mathrm{C}_{6} \mathrm{H}_{4}\right)_{3} \mathrm{CN}$ & $3.5(532 \mathrm{~nm})$ & 60 & $7.5(532 \mathrm{~nm})$ \\
& $6(586 \mathrm{~nm})$ & \multicolumn{2}{c}{$13(586 \mathrm{~nm})$} \\
\hline
\end{tabular}

Values are for 532-nm excitation, except for 5CT where excitation at 586 nm was also used.

${ }^{6}$ Molecules were spread at a surface density of $3 \times 10^{14}$ molecules $/ \mathrm{cm}^{2}(8 C B$ and $12 \mathrm{CB}$ ) or $2.5 \times 10^{14}$ molecules $/ \mathrm{cm}^{2}(8 \mathrm{BCA}, 7 \mathrm{CPP}$, and $5 \mathrm{CT})$.

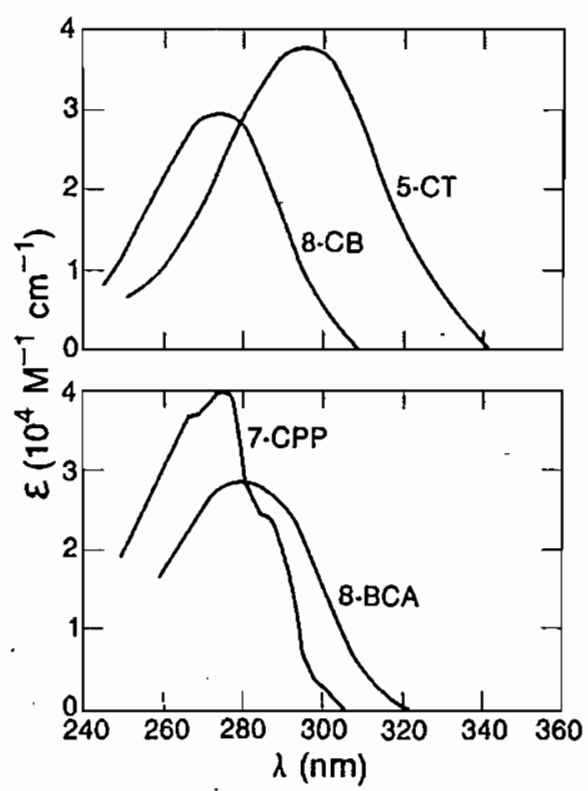

Fig. 5. Comparison of absorption spectra in $n$-hexane solutions.

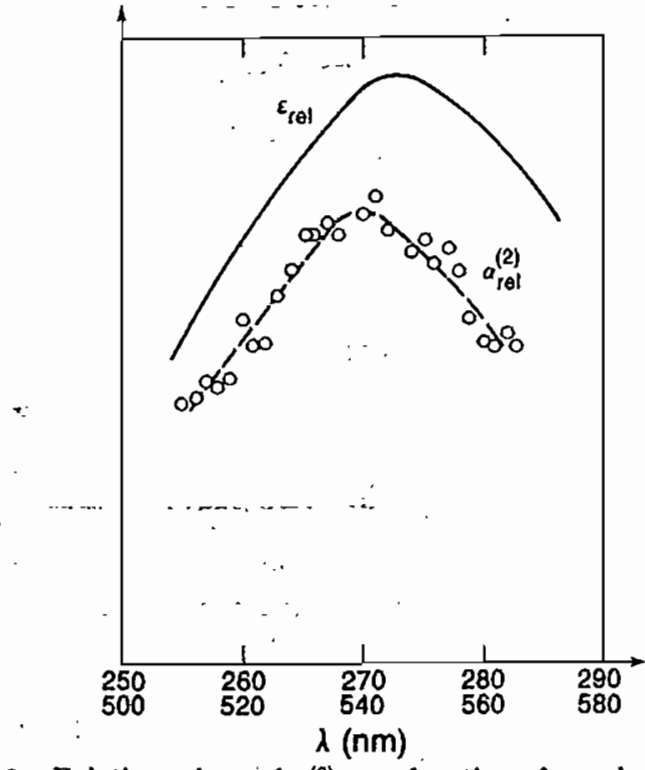

Fig. 6. Relative values of $\alpha^{(2)}$ as a function of wavelength for an $8 \mathrm{CB}$ monolayer on water are compared with its absorption spectrum in $n$-hexane. The top horizontal axis scale refers to absorption, and the bottom scale refers to $\alpha^{(2)}$

should also be measured near its $2 \omega$ resonance at $\sim 295 \mathrm{~nm}$. As is shown in Table $1, \alpha^{(2)}$ for 5CT increases when the excitation wavelength is changed from 532 to $586 \mathrm{~nm}$.

Resonant enhancement of $\alpha^{(2)}$ and SHG was also demonstrated for $8 C B$ when the excitation wavelength was varied between 510 and $560 \mathrm{~nm}$. Here, relative values of $\alpha^{(2)}$ were determined by using SHG from a crystalline quartz plate as a reference. Results are presented in Fig. 6. They show that the wavelength dependence of $\alpha^{(2)}$ follows that of the absorption spectrum of $8 \mathrm{CB}$ in $n$-hexane at $2 \omega$. The slight shift between the peaks could be due to the difference of the molecular environments in the two cases. This experiment therefore also demonstrates the potential of SHG for surface spectroscopy of adsorbate monolayers.

\section{DISCUSSION $\because, \cdots$}

The $\alpha^{(2)}$ values determined here are quite high, e.g., $\alpha^{(2)} \sim$ $2.5 \times 10^{-29}$ esu for 8CB compared with $\alpha^{(2)} \sim 4 \times 10^{-29} \mathrm{esu}$ for 2-methyl-4-nitroaniline (MNA) at $1.06 \mu \mathrm{m} .{ }^{14}$ The same value of $\alpha^{(2)}$ is observed for $8 \mathrm{CB}$ and $12 \mathrm{CB}$, indicating that the length of the hydrocarbon tail plays no role in $\alpha^{(2)}$. This is in agreement with our earlier results for $8,9,10$, and $12 \mathrm{CB}, 5$ although an EFISH study ${ }^{15}$ reported some differences in $\alpha^{(2)}$ for 5CB and 8CB. However, the cyano end group, which is more polar and a better electron donor than $\mathrm{COOH}$, adds considerably to $\alpha^{(2)}$, as evidenced by the much smaller value of $\alpha^{(2)}$ for 8BCA. There is also a significant decrease in $\alpha^{(2)}$ when $a$ phenyl ring is replaced by a pyrimidine ring, as shown by the smaller $\alpha^{(2)}$ value for 7CPP. This presumably arises from a significant interruption of electron delocalization in the pyrimidine ring. A surprising result is the decrease in $\alpha^{(2)}$ when a third phenyl ring is added as in 5CT. Although at first one might be tempted to think that $\alpha^{(2)}$ would increase as a result of a larger delocalized $\pi$ system, ${ }^{16}$ this is clearly not the case. Further investigation of this effect is needed.

Although not of primary interest in the present study, the 
monolayer method also indicates trends in the orientation of molecules at the water surface, as these experiments yield $\theta$, the average orientation of the major nonlinear optical axis (in this case the biphenyl long molecular axis ${ }^{5}$ ) with respect to the normal of the water surface (see Table 1). All molecules appear to orient with a large tilt angle, and the morewater-soluble 7CPP containing the pyrimidine ring has the largest $\theta$, whereas the molecule with the terphenyl ring is more vertical than the ones with the biphenyl ring. It should be pointed out that these conclusions regarding molecular orientations apply only to the biphenyl (or terphenyl) part of the molecule, to which the more flexible hydrocarbon chain need not be parallel.

\section{ACKNOWLEDGMENTS}

The authors thank R. Superfine and P. Guyot-Sionnest for assistance and helpful discussions and D. J. Gerbi, R. E. Harelstad, and M. S. Spiering (3M Research Laboratories) and M. Schadt (Hoffmann LaRoche) for gifts of materials. G. Berkovic acknowledges a Chaim Weizmann postdoctoral fellowship. This work was supported by the Director, Office of Energy Research, Office of Basic Energy Sciences, Materials Sciences Division of the U.S. Department of Energy under contract no. DE-AC03-76SF00098.

\section{REFERENCES}

1. J. Zyss, J. Mol. Electron. 1, 25-45 (1985).

2. D. J. Williams, Angew. Chem. Int. Ed. 23, 690-703 (1984),

3. S. K. Kurtz and T. T. Perry, J. Appl. Phys. 39, 3798-3813 (1968)

4. J. L. Oudar, J. Chem. Phys, 67, 446-457 (1977).

5. Th. Rasing, G. Berkovic, Y. R. Shen, S. G. Grubb, and M. W. Kim, Chem. Phys. Lett. 130, 1-5 (1986).

6. Y. R. Shen, J. Vac. Sci. Technol. B 3, 1464-1466 (1985), and references therein.

7. T. F. Heinz, C. K. Chen, D. Ricard, and Y. R. Shen, Phys. Rev. Lett. 48, 478-481 (1982).

8. T. F. Heinz, H. W. K. Tom, and Y. R. Shen, Phys. Rev. A 28, 1883-1885 (1983).

9. I. R. Girling, N. A. Cade, P. V. Kolinsky, and C. M. Montgomery, Electron. Lett. 21, 169-170 (1985).

10. I. R. Girling, N. A. Cade, P. V. Kolinsky, J. D. Earls, G. H. Cross, and I. R. Peterson, Thin Solid Films 132, 101-112 (1985).

11. P. Guyot-Sionnest, W. Chen, and Y. R. Shen, Phys. Rev. B 33, 8254-8263 (1986).

12. W. D. Harkins, The Physical Chemistry of Surface Films (Reinhold, New York, 1952).

13. G. M. Bell, L. C. Coombs, and L. J. Dunne, Chem. Rev. 81, 15-48 (1981).

14. B. F. Levine, C. G. Bethea, C. D. Thurmond, R. T. Lynch, and J. L. Bernstein, J. Appl. Phys. 50, 2523-2527 (1979).

15. M. I. Barnik, L. M. Blinov, A. M. Dorozhkin, and N. M. Shtykov, Sov. Phys. JETP 54, 935-938 (1981).

16. A. Dulcic, C. Flytzanis, C. L. Tang, D. Pepin, M. Fetizon, and Y. Hoppilliard, J. Chem. Phys. 74, 1559-1563 (1981). 